\section{Pour une production contrôlée d'agarwood d'Aquilaria crassna Pierre ex Lecomte en Guyane : approches métagénomique, biochimique et histologique}

Clara ZAREMSKI

\section{RÉSUMÉ}

Aquilaria Lam. est un genre d'arbre tropical de la famille des Thymelaeaceae dont le bois sain est de couleur blanche. Lorsque l'arbre est blessé, en interactions avec des micro-organismes environnementaux, l'arbre produit un bois transformé par sa forte teneur en composés secondaires : l'agarwood. L'agarwood, de couleur brun-noir, est particulièrement apprécié pour son odeur boisée intense. L'huile essentielle obtenue par hydrodistillation d'agarwood est très prisée en parfumerie. Ainsi, le marché mondial des produits dérivés d'Aquilaria est estimé, en 2018 , à 11 milliards de dollars US. Face à la demande croissante sur le marché mondial, des agriculteurs guyanais ont réalisé des plantations expérimentales d'Aquilaria en Guyane. Ainsi, l'objectif du projet Aquil@Guyane est d'aider ces planteurs à produire une huile essentielle de composition chimique contrôlée, respectueuse de la biologie de l'arbre et de l'environnement. Dans ce contexte, le sujet de thèse a été orienté en vue de promouvoir la production de connaissances académiques de nature à contribuer au développement de la production d'agarwood d'Aquilaria crassna en Guyane. Après caractérisation des communautés fongiques présentes dans les arbres d'Aquilaria crassna en Guyane, permettant de révéler l'importance des Basidiomycètes dans la production d'agarwood, deux méthodes d'induction d'agarwood ont été comparées : une méthode d'induction par blessure, avec ou sans ajout de terre ; une méthode d'induction par inoculation de souches guyanaises sélectionnées de Basidiomycètes. Cette dernière méthode, notamment par inoculation de champignons de pourriture fibreuse, comme Pycnoporus sanguineus, est la plus performante pour obtenir un agarwood de qualité proche d'un agarwood de référence provenant du Laos. La spectrométrie proche infrarouge (SPIR) a été utilisée pour différencier le bois blanc et l'agarwood et pour distinguer différentes compositions chimiques d'agarwood. Les résultats obtenus sont cohérents avec ceux de l'analyse biochimique après hydrodistillation : l'utilisation de la SPIR pour trier et contrôler la qualité de l'agarwood avant son hydrodistillation est envisageable. Enfin, une méthode de culture de cals d'Aquilaria a été mise au point afin de comprendre, en laboratoire, les mécanismes moléculaires qui aboutissent à la production d'agarwood. Les résultats indiquent une production de terpènes par les cals d'Aquilaria contaminés par des souches de champignons de pourriture fibreuse.

Mots-clés : agarwood, Aquilaria, cals, champignons, composition chimique, développement, histologie, huile essentielle, micro-organismes, spectrométrie proche infrarouge (SPIR), unités taxonomiques opérationnelles (UTO), Guyane.

\section{Developing controlled production of agarwood from Aquilaria crassna Pierre ex Lecomte in French Guiana: metagenomic, biochemical and histological approaches}

\section{ABSTRACT}

Aquilaria Lam. is a tropical tree genus of the Thymelaeaceae family whose wood when healthy is white in colour. When the tree is wounded, the wood it produces is transformed by interactions with surrounding micro-organisms that produce a high concentration of secondary components. The wood is then known as agarwood. It is blackish-brown in colour and prized for its intense woody fragrance. The essential oil obtained from agarwood by hydrodistillation is highly valued by the perfume industry. The world market for Aquilaria products was estimated in 2018 at 11 billion US\$. In response to rising demand on the world market, farmers in Guiana have been undertaking experimental planting of Aquilaria. The Aquil@Guyane project aims to help these farmers to produce essential oil with a chemically controlled composition using methods suited to the biology and environment of the species. Given this context, the subject of this thesis was geared to the production of academic knowledge that would contribute to the development of agarwood production from Aquilaria crassna in Guiana. Characterisation of the fungal communities present in Aquilaria crassna trees in Guiana revealed the important role of Basidiomycetes in the production of agarwood. Two methods for inducing agarwood formation were then compared: one involving induction by wounding, with or without added soil, and one involving induction by inoculation with selected Guianese strains of Basidiomycetes. The latter method, especially with inoculation of a white rot fungus, such as Pycnoporus sanguineus, proved most effective for obtaining agarwood of a quality close to that of a reference agarwood from Laos. Near-infrared spectrometry (NIRS) was used to differentiate white wood from agarwood and to distinguish between different chemical compositions of the agarwood. The results obtained are consistent with those obtained by biochemical analysis after hydrodistillation: the use of NIRS to sort the agarwood and check its quality before hydrodistillation can therefore be considered. Finally, a method for culturing Aquilaria calluses was developed in order to understand, in laboratory conditions, the molecular mechanisms that result in the production of agarwood. Our results indicate that Aquilaria calluses produce terpenes when contaminated by strains of white rot fungi.

Keywords: agarwood, Aquilaria, calluses, fungi, chemical composition, development, histology, essential oil, microorganisms, Near Infrared Spectrometry (NIRS), Operational Taxonomic Units (OTU), French Guiana.

\section{Producción controlada de madera de agar de Aquilaria crassna Pierre ex Lecomte en la Guayana Francesa: enfoque metagenómico, bioquímico e histológico}

\section{RESUMEN}

Aquilaria Lam. es un género de árbol tropical de la familia de las Thymelaeaceae cuya madera sana es de color blanco. Cuando está herido, al interaccionar con microorganismos ambientales, el árbol produce una madera transformada por una fuerte presencia de compuestos secundarios: la madera de agar o "Oud". La madera de agar, de color marrón negruzco, es particularmente apreciada por su intenso aroma a madera. El aceite esencial obtenido por hidrodestilación de la madera de agar es muy apreciado en perfumería. Así, el mercado mundial de los productos derivados de Aquilaria se estimó, el año 2018, en 11 mil millones de USD. Ante la demanda creciente en el mercado mundial, los agricultores guayaneses realizaron plantaciones experimentales de Aquila ria en la Guayana Francesa. El objetivo del proyecto Aquil@Guyane es ayudar a estos cultivadores a producir un aceite esencial de composición química controlada, que respete la biología del árbol y el medio ambiente. En este contexto, el tema de la tesis se orientó hacia la generación de conocimientos académicos que contribuyan al desarrollo de la producción de madera de agar de Aquilaria crassna en la Guayana Francesa. Después de la caracterización de las comunidades fúngicas presentes en los árboles de Aquilaria crassna de la Guayana Francesa, que reveló la importancia de los basidiomicetos en la producción de madera de agar, se compararon dos métodos de inducción de madera de agar: un método de inducción por herida, añadiendo o sin añadir tierra, y un método de inducción por inoculación de cepas guyanesas seleccionadas de basidiomicetos. Este último método, especialmente con la inoculación de hongos de podredumbre fibrosa, como el Pycnoporus sanguineus, es el más eficaz para obtener una madera de agar de calidad cercana a una madera de agar de referencia proveniente de Laos. La espectrometría del infrarrojo próximo (NIRS) fue utilizada para diferenciar la madera blanca y la madera de agar, y para distinguir diferentes composiciones químicas de la madera de agar. Los resultados obtenidos son coherentes con los del análisis bioquímico después de la hidrodestilación: la utilización de la NIRS para clasificar y controlar la calidad de la madera de agar antes de su hidrodestilación es factible. En conclusión, se ha preparado un método de cultivo de callos de Aquilaria para comprender, en laboratorio, los mecanismos moleculares que conducen a la producción de madera de agar. Los resultados indican que hay producción de terpenos en los callos de Aquilaria contaminados por cepas de hongos de podredumbre fibrosa.

Mots-clés : madera de agar, Aquilaria, callos, hongos, composición química, desarrollo, histología, aceite esencial, microorganismos, espectrometría del infrarrojo próximo (NIRS), unidades taxonómicas operacionales (UTO), Guayana Francesa.

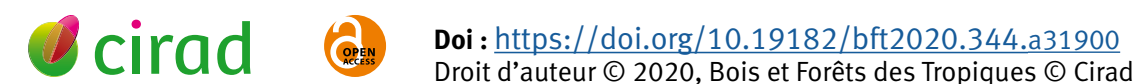
Date de publication : juillet 2020 


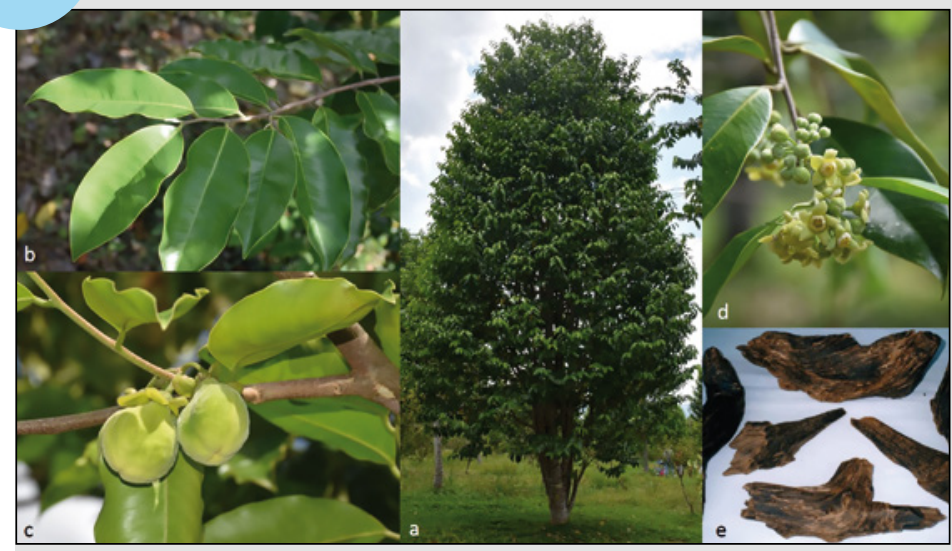

Photos

Arbre d'Aquilaria crassna, âgé de 5 ans, sur un champ du bourg de Cacao en Guyane (a). Les feuilles sont de forme elliptique (b). Les fruits sont des capsules à deux valves mesurant 3-5 x 2-4 cm, contenant une à deux graines (c). Les fleurs sont petites et jaunes, groupées en inflorescences axillaires en ombelles veloutées (d). Plus l'agarwood est sombre et ancien, plus sa valeur ajoutée est grande (e).

\section{Photos}

A 5 year-old Aquilaria crassna tree in a field near the village of Cacao in French Guiana (a). The leaves are elliptical in shape (b). The fruits are bivalve capsules measuring $3-5 \times 2-4 \mathrm{~cm}$ and containing one or two seeds (c). The small yellow flowers form velvety umbels grouped into axillary inflorescences (d). The older and darker the agarwood, the greater its added value (e)

\section{Fotos}

Árbol de Aquilaria crassna, 5 años de edad, en un campo de la población de Cacao, en la Guayana Francesa (a). Las hojas tienen forma elíptica (b). Las frutas son cápsulas bivalvas que miden $3-5 \times 2-4 \mathrm{~cm}$, y contienen de una a dos semillas (c). Las flores son pequeñas y amarillas, agrupadas en inflorescencias axilares en umbelas aterciopeladas (d). Como más oscura y vieja sea la madera de agar, mayor es su valor añadido (e).

\section{Publications}

Prix

Ron Cockcroft Award of International Research Group on Wood Protection, IRG 52, Numazu City, Japan, 9-13 May 2021. Zaremski C., Ducousso M., Andary C., et al., 2020. Chemical composition of agarwood of Aquilaria crassna Pierre ex. Lecomte induced by Basidiomycetes from French Guiana.

\section{Articles publiés}

Zaremski C, Ducousso-Détrez A., Amusant N., et al., 2019. Taxonomic study of French Guiana fungi to identify and isolate pure cultured fungi for oleoresin production in Aquilaria: use of sequences from the small ribosomal DNA ( $r$ ) subunit (SSU) and the two primer pairs SR6/SR10R and SR7/SR1R. PRO Ligno, 15 (3): 3-15. http://www.proligno.ro/en/articles/2019/3/ZAREMSKI.pdf

Zaremski C., Malandain C., Sibourg O., et al., 2018. NGS identification of fungi potentially implicated in the production of agarwood from Aquilaria spp. trees. PRO Ligno, 14 (3): 9-18. http://www.proligno.ro/en/articles/2018/3/ZREMSKI.pdf

\section{Article soumis}

Zaremski C., Andary C., Amusant N., et al., 2019. Review: A review of the conditions of worldwide agarwood production and development prospects in French Guiana. Biodiversity and Conservation, $36 \mathrm{p}$. [Sumitted]

\section{Grade et diplôme : Doctorat.}

Université : Université de Guyane, France.

Date de soutenance : 4 février 2020.

\section{Composition du jury}

Direction : Marc DUCOUSSO (Directeur de recherche, Cirad-LSTM, Hérault, France)

Encadrement : Nadine AMUSANT (Chercheur, Cirad-EcoFoG, Guyane, France).

Membres : Amadou BÂ (Professeur des Universités, Université des Antilles, Guadeloupe, France).

Christine GÉRARDIN (Professeur des Universités, Université de Lorraine, Lorraine, France).

Jacques BEAUCHÊNE (Chercheur, Cirad-EcoFoG, Guyane, France). Marie FLEURY (Maître de conférences, Muséum national d'Histoire naturelle, Guyane, France).

Franck ROUBAUD (Maître de conférences, Université de Guyane, Guyane, France)

Langue de rédaction : français

\section{Contact}

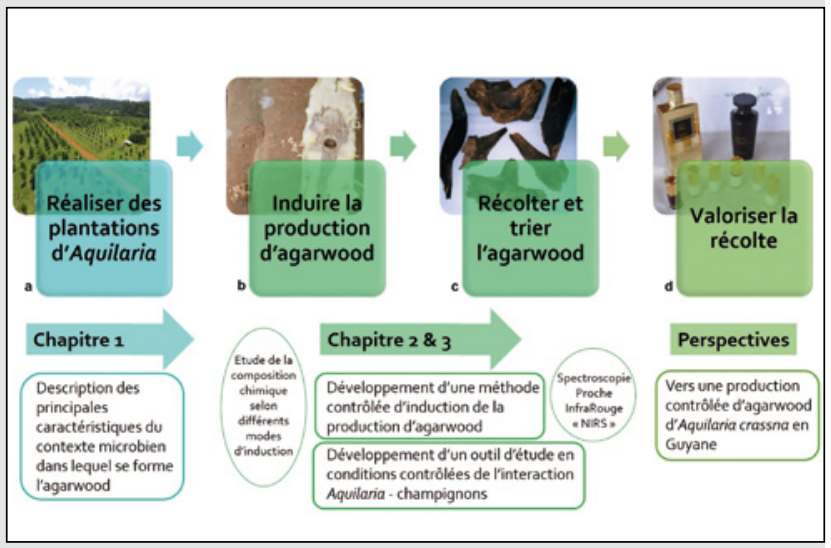

Figure

Les principales étapes de la production d'agarwood ont été le fil directeur des travaux de thèse divisés en trois chapitres. La description des principales caractéristiques du contexte microbien dans lequel se forme l'agarwood (chapitre 1) a mis en évidence l'importance des Basidiomycètes dans la transformation du bois d'Aquilaria crassna en agarwood. Ces résultats ont permis de développer une méthode contrôlée d'induction de la production d'agarwood à partir de souches guyanaises de champignons lignivores Basidiomycètes (chapitre 2) dont certaines souches de pourritures fibreuses ont montré les meilleurs résultats. Ces travaux ont été réalisés en champ et au laboratoire par l'étude, après hydrodistillation et mesure en SPIR, de la composition chimique de l'agarwood obtenue par les différents modes d'induction. Ils ont été couplés à une étude en laboratoire qui a permis de développer un outil d'étude en conditions contrôlées de l'interaction Aquilaria champignons (chapitre 3). L'objectif de ce travail est de permettre aux agriculteurs de Guyane, planteurs d'A. crassna, de développer une production contrôlée d'agarwood en Guyane.

\section{Figure}

The main stages in agarwood production provided the structure for the work on this thesis, which is divided into three chapters. Chapter 1 describes the main characteristics of the microbial context in which agarwood forms and shows the important role of Basidiomycetes in the transformation of Aquilaria crassna wood into agarwood. From these results, we were able to develop a controlled method for inducing the production of agarwood using Guianese strains of Basidiomycetes wood-decay fungi (Chapter 2), with some strains of white rot fungi producing the best results. These studies were conducted in the field and in the laboratory, by analysing, after hydrodistillation and NIRS measurements, the chemical composition of the agarwood obtained with different induction methods. The studies were combined with laboratory work resulting in the development of a tool for analysing Aquilaria - fungi interactions under controlled conditions (Chapter 3). The aim of the research work is to enable Guianese farmers planting $A$. crassna trees to develop controlled production of agarwood in French Guiana Figure legends: (a) Establishing Aquilaria plantations. (b) Inducing the formation of agarwood. (c) Harvesting and sorting the agarwood produced. (d) Commercialising the harvest.

\section{Figura}

Las principales etapas de la producción de madera de agar fueron el hilo conductor del trabajo de tesis, dividido en tres capítulos. La descripción de las principales características del contexto microbiano en el que se forma la madera de agar (capítulo 1) puso en evidencia la importancia de los basidiomicetos en la transformación de la madera de Aquilaria crassna en madera de agar. Estos resultados permitieron desarrollar un método controlado de inducción de la producción de madera de agar a partir de cepas guyanesas de hongos lignívoros basidiomicetos (capítulo 2); entre las mismas, ciertas cepas de podredumbre fibrosa proporcionaron los mejores resultados. Estos ensayos se realizaron en el campo y en el laboratorio para, después de la hidrodestilación y medición en NIRS, estudiar la composición química de la madera de agar obtenida mediante los diferentes métodos de inducción. Se asociaron a un estudio de laboratorio que permitió desarrollar una herramienta de investigación en condiciones controladas de la interacción Aquilaria - hongos (capítulo 3). El objetivo de este trabajo es permitir a los agricultores de la Guayana Francesa, cultivadores de $A$. crassna, desarrollar una producción controlada de madera de agar en la Guayana Francesa.

Textos de la figura: (a) Plantación de Aquilaria. (b) Inducción de producción de madera de agar. (c) Cosecha y clasificación de la madera de agar. (d) Valorización de la cosecha. 